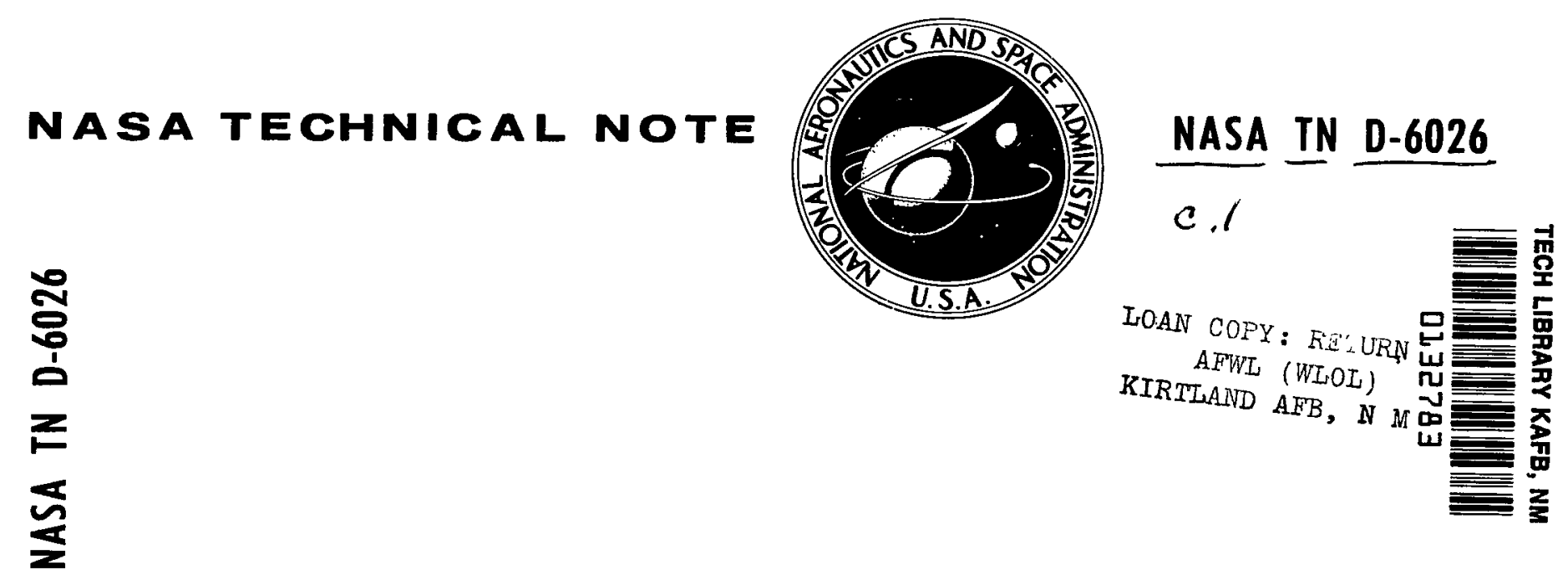

\title{
GROWTH DUE TO BUOYANCY OF WEAK HOMOGENEOUS TURBULENCE WITH SHEAR
}

by Robert G. Deissler

Lewis Research Center Cleveland, Ohio 44135 


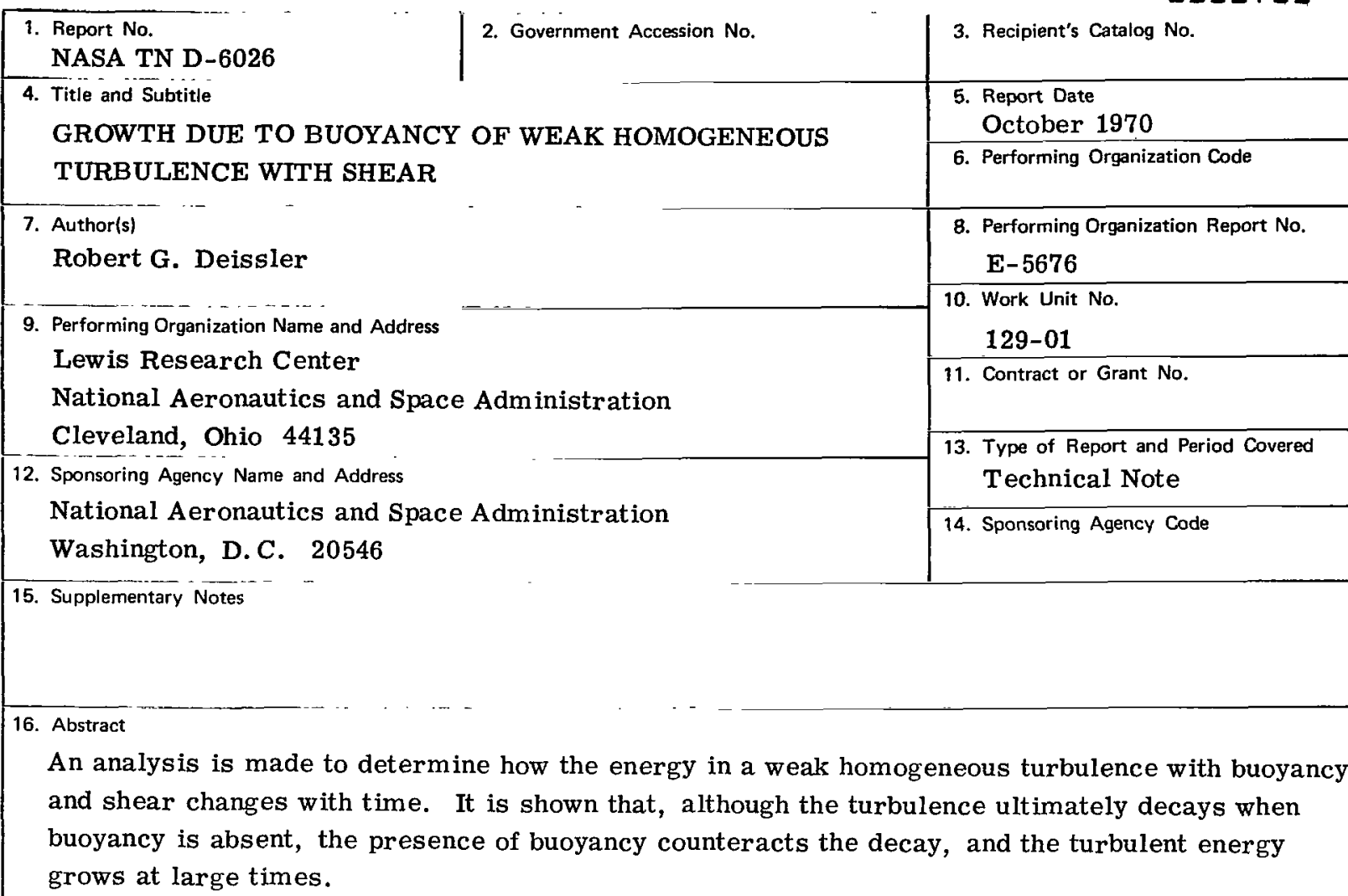

17. Key Words (Suggested by Author(s))

Turbulence

Homogeneous

Buoyancy
19. Security Classif. (of this report) Unclassified
Shear

Growth
18. Distribution Statement

Unclassified - unlimited

\begin{tabular}{|c|c|c|}
\hline \multicolumn{3}{|c|}{$\mid \begin{array}{l}\text { 18. } \text { Distribution Statement } \\
\text { Unclassified - unlimited }\end{array}$} \\
\hline $\begin{array}{l}\text { of this page) } \\
\text { ified }\end{array}$ & $\begin{array}{c}\text { 21. No. of Pages } \\
14\end{array}$ & $\begin{array}{l}\text { 22. Price }{ }^{*} \\
\$ 3.00\end{array}$ \\
\hline
\end{tabular}

"For sale by the Clearinghouse for Federal Scientific and Technical Information Soringfield. Virainia 22151 


\title{
GROWTH DUE TO BUOYANCY OF WEAK HOMOGENEOUS TURBULENCE WITH SHEAR
}

\author{
by Robert G. Deissler \\ Lewis Research Center
}

\section{SUMMARY}

An analysis is made to determine how the energy in a weak homogeneous turbulence with buoyancy and shear changes with time. It is shown that, although the turbulence ultimately decays when buoyancy is absent, the presence of buoyancy counteracts the decay, and the turbulent energy grows at large times.

\section{INTRODUCTION}

Studies of weak homogeneous (grid generated) turbulence with uniform mean velocity gradient are given in references 1 and 2 . In the results obtained there, the turbulent energy always decayed with time. The energy produced by the mean velocity gradient was at all times less than that dissipated. In reference 3 , where the initial condition was modified to give a finite initial turbulent energy, the energy sometimes increased for a while, but it still ultimately decayed. This behavior was attributed to the fact that, while the total energy was increasing, energy was being drained out of the component of the turbulence in the direction of the velocity gradient by the pressure-velocity correlations. Since there was no turbulence production in that component, it quickly decayed, with the result that the turbulent shear stress, and consequently the turbulence production in all the components, ultimately decreased.

Thus, the key to obtaining a nondecaying turbulent shear flow appears to lie in keeping the energy from being drained out of the turbulence component in the direction of the mean velocity gradient. In strong turbulence, the distribution of energy among the directional components is evidently accomplished by the pressure-velocity correlations, but in weak turbulence, those correlations generally tend to make the turbulence more anisotropic (ref. 1). If, however, we superimpose on the shear flow destabilizing buoyancy forces in the direction of the mean velocity gradient, it may be possible to 
obtain a nondecaying solution, even for weak turbulence. Those buoyancy forces should tend to prevent the turbulence component in the direction of the mean velocity gradient from decaying.

Some of the effects of combined buoyancy and shear on weak homogeneous turbulence were investigated in reference 4. However, the question of whether or not the turbulence decays was not considered. It is with that aspect of the problem that we will be concerned here.

\section{ANALYSIS}

The basic equations for the present study can be written as (ref. 4)

$$
\begin{gathered}
\frac{\partial u_{i}}{\partial t}+u_{k} \frac{\partial u_{i}}{\partial x_{k}}+U_{k} \frac{\partial u_{i}}{\partial x_{k}}+\frac{\partial\left(u_{i} u_{k}\right)}{\partial x_{k}}-\frac{\partial \overline{u_{i} u_{k}}}{\partial x_{k}}=-\frac{1}{\rho} \frac{\partial\left(p-p_{e}\right)}{\partial x_{i}}+\nu \frac{\partial^{2} u_{i}}{\partial x_{k} \partial x_{k}}-\beta g_{i} \tau \\
\frac{1}{\rho} \frac{\partial^{2}\left(p-p_{e}\right)}{\partial x_{i} \partial x_{i}}=-2 \frac{\partial u_{i}}{\partial x_{k}} \frac{\partial u_{k}}{\partial x_{i}}-\frac{\partial^{2}\left(u_{i} u_{k}\right)}{\partial x_{i} \partial x_{k}}+\frac{\partial^{2} \overline{u_{i} u_{k}}}{\partial x_{i} \partial x_{k}}-\beta g_{i} \frac{\partial \tau}{\partial x_{i}}
\end{gathered}
$$

and

$$
\frac{\partial \tau}{\partial \mathrm{t}}+\mathrm{u}_{\mathrm{k}} \frac{\partial \mathrm{T}}{\partial \mathrm{x}_{\mathrm{k}}}+\mathrm{U}_{\mathrm{k}} \frac{\partial \tau}{\partial \mathrm{x}_{\mathrm{k}}}+\frac{\partial\left(\tau \mathrm{u}_{\mathrm{k}}\right)}{\partial \mathrm{x}_{\mathrm{k}}}-\frac{\partial \overline{\tau \mathrm{u}_{\mathrm{k}}}}{\partial \mathrm{x}_{\mathrm{k}}}=\alpha \frac{\partial^{2} \tau}{\partial \mathrm{x}_{\mathrm{k}} \partial \mathrm{x}_{\mathrm{k}}}
$$

where the subscripts (except e) can take on the values 1,2 , or 3 , and a repeated subscript in a term signifies a summation. The quantity $u_{i}$ is a fluctuating velocity component, $\mathrm{t}$ is the time, $\mathrm{U}_{\mathrm{i}}$ is a mean velocity component, $\rho$ is the density, $\mathrm{p}$ is the instantaneous pressure, $\mathrm{p}_{\mathrm{e}}$ is the equilibrium pressure, $\mathrm{x}_{\mathrm{i}}$ is a space coordinate, $\nu$ is the kinematic viscosity, $\beta=-(1 / \rho)(\partial \rho / \partial \mathrm{T}) \mathrm{p}, \mathrm{g}_{\mathrm{i}}$ is a component of the body force per unit mass, $\tau$ is the temperature fluctuation, $T$ is the mean temperature, $\alpha$ is the thermal diffusivity, and overbars designate averaged values. (Symbols are defined in the appendix.)

In obtaining equations (1) to (3), the instantaneous velocities and temperatures in the incompressible Navier-Stokes and energy equations are broken into mean and fluctuating components. For the last term in equation (1) (buoyancy term), the density is 
assumed to depend effectively only on temperature and is not far removed from its equilibrium value (value it would have for no heat transfer or turbulence). The equation for the pressure (eq. (2)) is obtained by taking the divergence of the Navier-Stokes equation and applying continuity.

Equations involving correlations between fluctuating quantities at two points $\mathbf{P}$ and $\mathrm{P}^{\prime}$ can be constructed from equations (1) to (3). Such equations have been obtained for homogeneous turbulence with uniform velocity and temperature gradients in reference 4. They can be converted to spectral form by taking their Fourier transforms. We consider the case where the velocity and temperature gradients are in the $\mathrm{x}_{3}$-direction (vertical) and the body force (gravity) is in the $-\mathrm{x}_{3}$-direction. Let $\mathrm{g}=-\mathrm{g}_{3}, \mathrm{a}=\mathrm{dU_{1 }} / \mathrm{dx}_{3}$, and $b=d T / d_{3}$, and assume that the turbulence is weak enough to neglect terms containing triple correlations. The correlation equations then become in spectral form

$$
\begin{aligned}
& \frac{\partial \varphi_{\mathrm{ij}}}{\partial \mathrm{t}}-\mathrm{a} \kappa_{1} \frac{\partial \varphi_{\mathrm{ij}}}{\partial \kappa_{3}}=-\mathrm{a}\left(\delta_{\mathrm{i} 1} \varphi_{\mathrm{j} 3}+\delta_{1 \mathrm{j}} \varphi_{\mathrm{i} 3}\right)+2 \mathrm{a}\left(\frac{\kappa_{1} \kappa_{\mathrm{j}}}{\kappa^{2}} \varphi_{\mathrm{i} 3}+\frac{\kappa_{\mathrm{i}} \kappa_{1}}{\kappa^{2}} \varphi_{\mathrm{j} 3}\right)+\beta g \gamma_{\mathrm{i}}\left(\delta_{\mathrm{j} 3}-\frac{\kappa_{3} \kappa_{\mathrm{j}}}{\kappa^{2}}\right) \\
& +\beta g \gamma_{j}\left(\delta_{i 3}-\frac{\kappa_{i} \kappa_{3}}{\kappa^{2}}\right)-2 \nu \kappa^{2} \varphi_{i j} \\
& \frac{\partial \gamma_{\mathbf{i}}}{\partial \mathrm{t}}-\mathrm{a} \kappa_{1} \frac{\partial \gamma_{\mathbf{i}}}{\partial \kappa_{3}}=-\mathrm{b} \varphi_{\mathrm{i} 3}+\mathrm{a} \gamma_{3}\left(2 \frac{\kappa_{\mathrm{i}} \kappa_{1}}{\kappa^{2}}-\delta_{\mathrm{i} 1}\right)+\beta \mathrm{g} \delta\left(\delta_{\mathrm{i} 3}-\frac{\kappa_{\mathrm{i}} \kappa_{3}}{\kappa^{2}}\right)-(\alpha+\nu) \kappa^{2} \gamma_{\mathrm{i}} \\
& \frac{\partial \delta}{\partial \mathrm{t}}-\mathrm{a} \kappa_{1} \frac{\partial \delta}{\partial \kappa_{3}}=-2 \mathrm{~b} \gamma_{3}-2 \alpha \kappa^{2} \delta
\end{aligned}
$$

where $\varphi_{i j}, \gamma_{i}$, and $\delta$ are given by

$$
\begin{aligned}
& \overline{u_{i} u_{j}^{\prime}}=\int_{-\infty}^{\infty} \varphi_{i j} e^{i \vec{i} \cdot \vec{r}} d \vec{\kappa} \\
& \overline{\tau u_{i}^{\prime}}=\int_{-\infty}^{\infty} \gamma_{i} e^{i \vec{\kappa} \cdot \vec{r}} d \vec{\kappa} \\
& \overline{\tau \tau^{\prime}}=\int_{-\infty}^{\infty} \delta \mathrm{e}^{i \vec{k} \cdot \vec{r}} d \vec{\kappa}
\end{aligned}
$$


The quantity $\vec{\kappa}$ is the wave number vector and $d \vec{\kappa}=d \kappa_{1} d \kappa_{2} d \kappa_{3}$. The magnitude of $\vec{\kappa}$ has the dimension $1 /$ length and can be considered to be the reciprocal of a wavelength or eddy size. The unprimed and primed quantities in the barred products refer, respectively, to values at points $P$ and $P^{\prime}$ separated by the vector $r$. The quantity $\delta_{i j}$ is the Kronecker delta.

Equations (4), (5), and (6) give contributions of various processes to the rate of change of spectral components of $\overline{u_{i} u_{j}}, \overline{\tau u_{i}}$, and $\bar{\tau}{ }^{2}$, respectively. The second term in each equation is a transfer term which transfers activity into or out of a spectral component by the stretching or compressing of turbulent vortex filaments by the mean velocity gradient, as discussed in reference 1 . The terms with $\kappa^{2}$ in the denominator are spectral components of pressure-velocity or pressure-temperature correlations and transfer activity between directional components (ref. 1). The terms proportional to $\beta \mathrm{g}$ and $\delta_{\mathrm{i} 3}$ (or $\delta_{\mathrm{j} 3}$ ) are buoyancy terms which augment or diminish the activity in a spectral component by buoyant action. The last terms in the equations are dissipation terms, which dissipate activity by viscous or by conduction effects. The remaining terms in the equations produce activity by velocity or temperature gradient effects.

For solving equations (4) to (6), the turbulence is assumed to be initially isotropic at $\mathrm{t}=\mathrm{t}_{0}$. That condition is satisfied by

$$
\varphi_{\mathrm{ij}}=\frac{\mathrm{J}_{0}}{12 \pi^{2}}\left(\kappa^{2} \delta_{\mathrm{ij}}-\kappa_{\mathrm{i}} \kappa_{\mathrm{j}}\right) \mathrm{e}^{-\left(\kappa / \kappa_{0}\right)^{2}}
$$

where $J_{0}$ is a constant that depends on initial conditions and $\kappa_{0}$ is an initial wave number that is characteristic of the turbulence. Equation (10) differs from the initial condition in references 1,2 , and 4 (but not from that in 3 ) by the exponential factor, which was set equal to 1 in those references $\left(\kappa_{0}=\infty\right)$. For the initial conditions on $\delta$ and $\gamma_{i}\left(\right.$ at $\left.t=t_{0}\right)$, it is assumed that

$$
\delta_{0}=\left(\gamma_{i}\right)_{0}=0
$$

That is, the turbulence producer (grid) is assumed to be unheated, so that the temperature fluctuations are produced by the interactions of the mean temperature gradient with the turbulence.

A method of solving the preceding set of partial differential equations (eqs. (4) to (6)) is described in reference 4 . Those equations are converted to ordinary differential equations, and spherical coordinates are introduced by using the transformations 
$\kappa_{1}=\kappa \cos \varphi \sin \theta, \kappa_{2}=\kappa \sin \varphi \sin \theta$, and $\kappa_{3}=\kappa \cos \theta$. The resulting set of equations is integrated numerically by machine computation. Directionally integrated spectrum functions can then be obtained from

$$
\left[\begin{array}{l}
\psi_{\mathrm{ij}} \\
\Gamma_{\mathrm{i}} \\
\Delta
\end{array}\right]=\int_{0}^{\pi} \int_{0}^{2 \pi}\left[\begin{array}{l}
\sigma_{\mathrm{ij}} \\
\gamma_{\mathrm{i}} \\
\delta
\end{array}\right] \kappa^{2} \sin \theta \mathrm{d} \varphi \mathrm{d} \theta
$$

These spectrum functions can be integrated over all wave numbers to give the following single-point correlations:

$$
\left[\begin{array}{c}
\overline{u_{i} u_{j}} \\
\overline{\tau u_{i}} \\
\overline{\tau^{2}}
\end{array}\right]=\int_{0}^{\infty}\left[\begin{array}{l}
\psi_{i j} \\
\Gamma_{i} \\
\Delta
\end{array}\right] d \kappa
$$

Computed correlations and spectra for the case where the buoyancy forces are destabilizing (negative vertical temperature gradients) will be considered in the next section. The results given there are for a gas with a Prandtl number of 0.7 .

\section{RESULTS AND DISCUSSION}

The effect of destabilizing buoyancy forces on weak homogeneous shear-flow turbulence in a gas is illustrated in figure 1. The superscript (a) on $\overline{u_{i} u_{j}}(a), t(a)$, and $\kappa_{0}^{(a)}$ indicates that those parameters have been made dimensionless by using quantities related to the shear (in contrast to those related to the buoyancy, which will be used later). Curves are shown for two values of Richardson number and of the initial wave number parameter.

The curves indicate that for a Richardson number of 0 (no buoyancy effects) all components of the turbulent energy decrease with time. The turbulent shear stress $\overline{-u_{1} u_{3}}$ also decreases with time, except near the initial time. (At $t^{(a)}=0$ the turbulence is isotropic and $\overline{\mathrm{u}_{1} \mathrm{u}_{3}}$ is 0. ) 


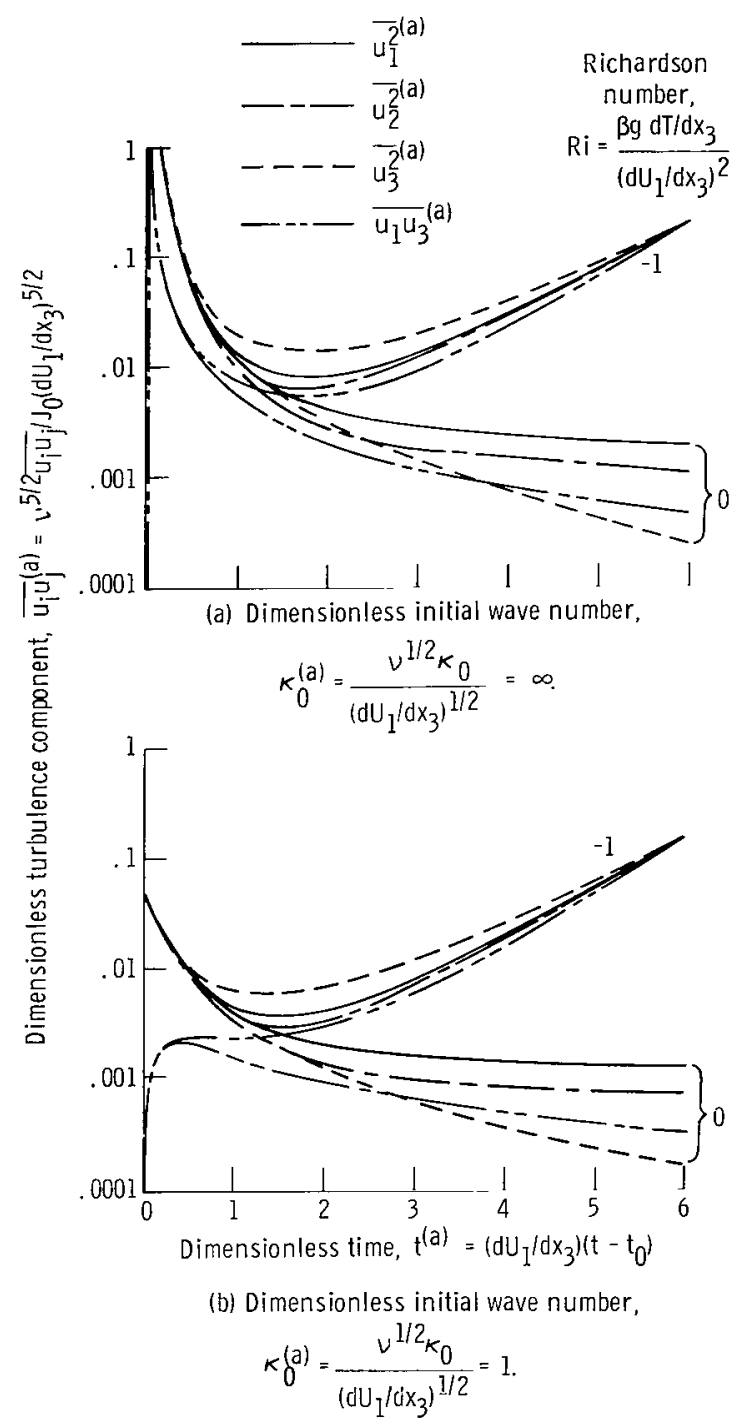

Figure 1. - Effect of destabilizing buoyancy on variation with time of weak turbulence in uniform uniform shear flow. Prandtl number, 0.7.

The decay of the components of turbulent energy for no buoyancy effects evidently occurs mainly because there is no production term in the equation for $\overline{u_{3} u_{3}}$ (component in the direction of the mean velocity gradient). This can be seen by letting $i=j=3$ in equation (4), in which case the production term (first term on the right side) drops out. In addition, the pressure-velocity correlation terms in equation (4) tend to drain energy out of the $\overline{u_{3}^{2}}$-component when the turbulence is weak, as discussed in reference 1 . As a result the $\mathrm{u}_{3}^{2}$-component decays rapidly compared with the other components, which have energy fed into them by the mean velocity gradient or by the pressure-velocity correlations (see fig. 1). When $\overline{u_{3}^{2}}$ decays, the shear component $\overline{u_{3} u_{1}}$ must also de- 
cay. There is then no mechanism for maintaining the turbulence since that maintenance apparently takes place as a result of work done on the turbulent shear stress by the velocity gradient. (See first term on right side of eq. (4).) All of the turbulence components must then decay.

By contrast, for $\mathrm{Ri}=-1$ (buoyancy forces destabilizing), all components of the turbulent energy decay for a while and then begin to increase without limit as time becomes large. This increase evidently occurs because the vertical buoyancy forces excite the $\overline{\mathrm{u}_{3}^{2}}$-component of the turbulence and replenish the energy being drained out of it.

It might seem surprising that all components of the turbulence continue to increase with time rather than level off. There are no boundaries on the flow considered here however, so that the effective Reynolds number and Rayleigh number of the mean flow are infinite. As the scale or mixing length of the turbulence continues to grow, the eddies encounter larger and larger velocity and temperature differences, so that the effective driving forces on the turbulence continue to grow.

Comparison of figures $1(a)$ and (b) shows, as expected, that for $\kappa_{0}^{(a)}=\infty$ (all wave numbers present), the components of the initial energy are infinite, whereas for $\kappa_{0}^{(a)}=1$ they have a finite value. The turbulent shear stress $-\overline{\mathrm{u}_{3} \mathrm{u}_{1}}$ starts at zero on both plots since the turbulent shear stress for isotropic turbulence is zero. For the case of $\kappa_{0}^{(a)}=\infty$, however, the value of $-\overline{u_{3} u_{1}}$ jumps to infinity in an infinitely short time and then decreases. For $\kappa_{0}^{(a)}=1,\left(-\frac{3}{u_{3} u_{1}}\right)$ first increases steadily and then either decreases $(\mathrm{Ri}=0)$ or continues to increase $(\mathrm{Ri}=-1)$.

To give an idea of the distribution of the turbulent energy with wave number, energy spectra (spectra of $\overline{u_{i} u_{i}}$ ) are plotted in figure 2 for $\kappa_{0}^{(a)}=\infty$ and $R i=0$ and -1 . For $t^{(a)}=0, \psi_{i i}$ is proportional to $\kappa^{4}$ (eq. (10)). As time increases, the spectra move to the smaller wave number regions; that is, the scale of the turbulence grows indefinitely large with time, since the fluid is unbounded.

Thus far we have been considering the effect of buoyancy on a shear-flow turbulence. Next we want to consider the related problem of the effect of imposing a mean shear on turbulence that is buoyancy-controlled. For doing this it is convenient to use the parameters $\overline{u_{i}^{2}}(b), t(b), \kappa_{0}^{(b)}, \overline{\tau^{2}}(b)$, and $\overline{\tau u_{3}}(b)$, which have been made dimensionless by using quantities related to the buoyancy (see figs. 3 and 4). The parameters used in figure 1 were, on the other hand, nondimensionalized by using quantities related to the shear.

The effects of shear on buoyancy-controlled turbulence are illustrated in figure 3 and 4, where $\overline{u_{i}^{2}}(\mathrm{~b}), \overline{\tau^{2}}(\mathrm{~b})$, and $\overline{\tau u_{3}}(\mathrm{~b})$ are plotted against $\mathrm{t}^{(\mathrm{b})}$ for several values of Richardson number and $\kappa_{0}^{(b)}$. For the case of no shear $(\mathrm{Ri}=-\infty)$ the results were obtained from the integrated equations in reference 5. All components of the turbulent energy, as well as the temperature fluctuations and the temperature-velocity correlations, increase as $t^{(b)}$ becomes large. This occurs even when shear is absent and the 


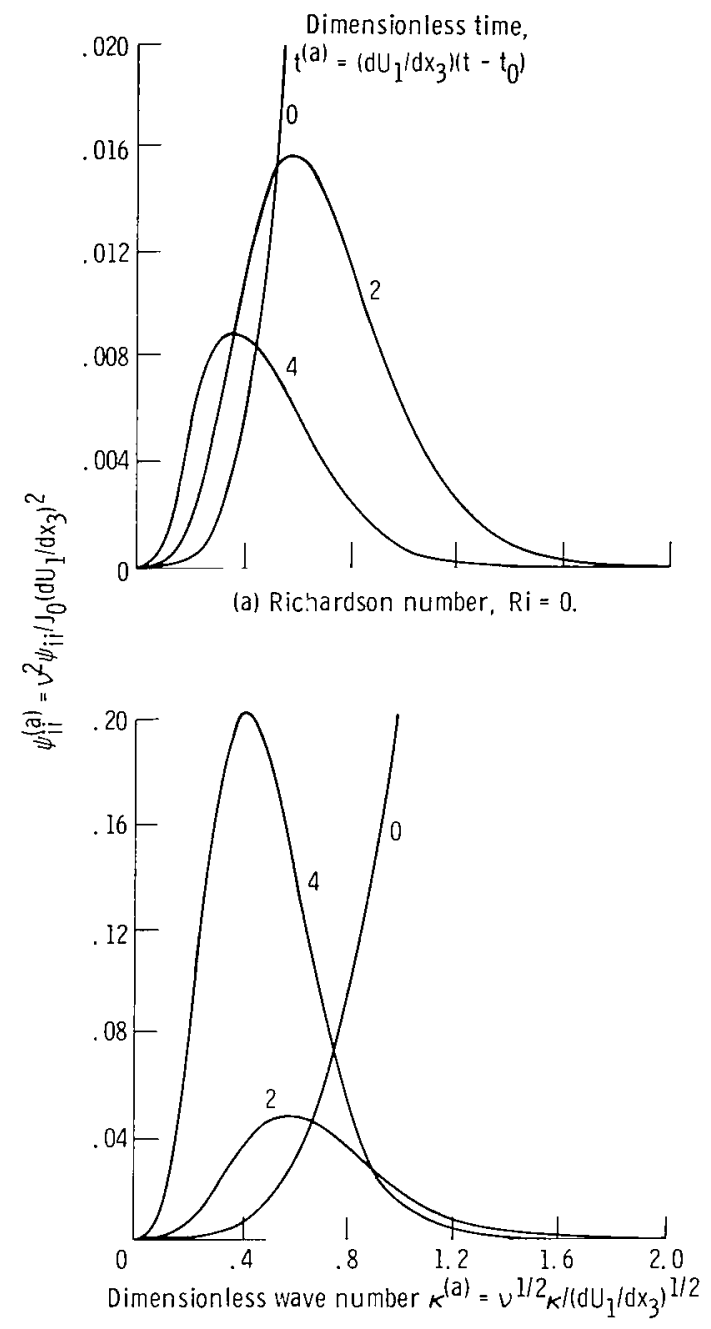

(b) Ricinardson number, $\mathrm{Ri}=-1$.

Figure 2. - Variation with time of turbulent energy spectra (spectra of $\overline{u_{i} u_{i}}$ ). Prandtl number, 0.7 . 


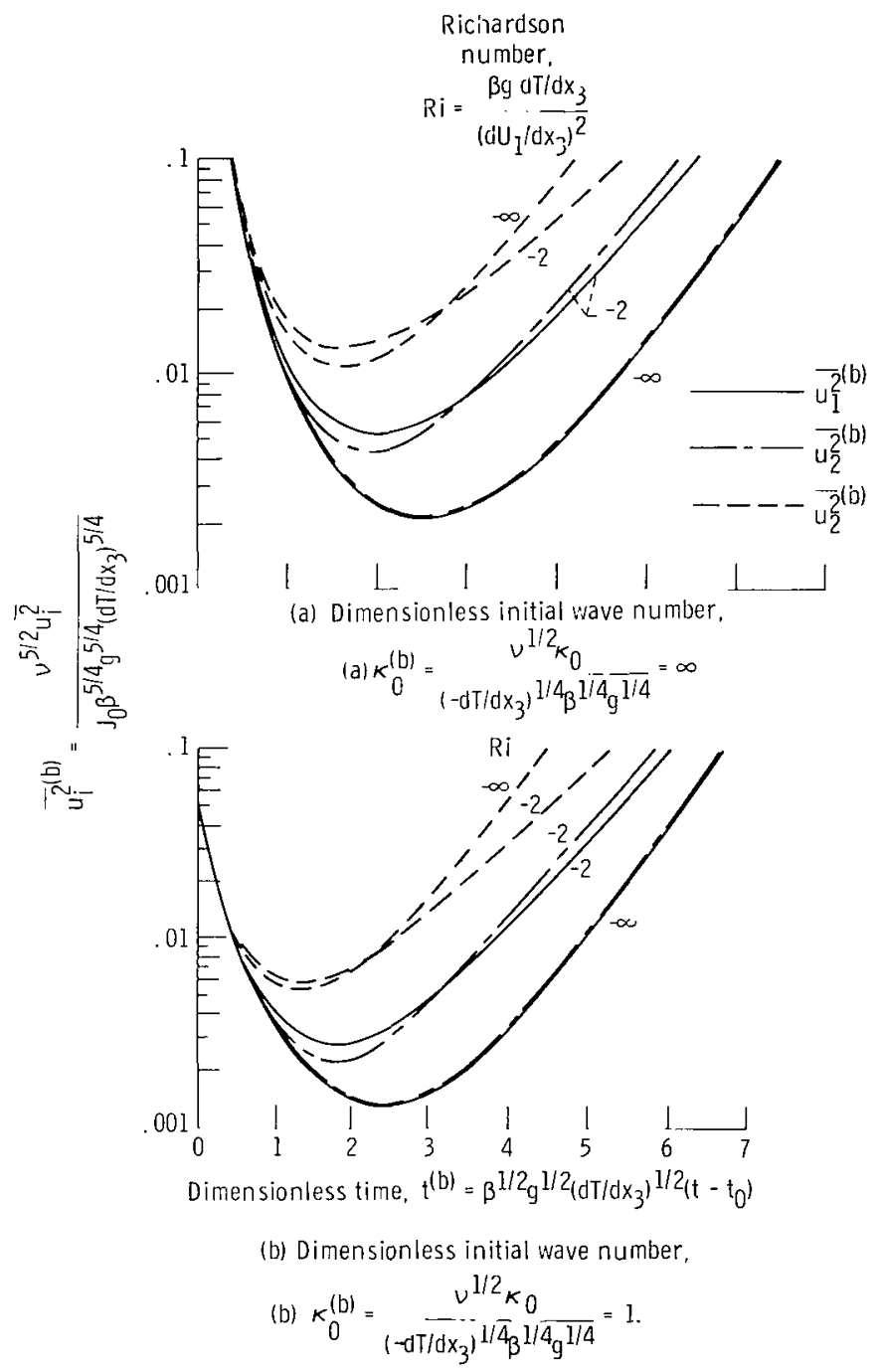

Figure 3. - Effect of uniform shear on variation with time of weak turbulence in flow with destabilizing buoyancy. Prandt! number, 0.7. 


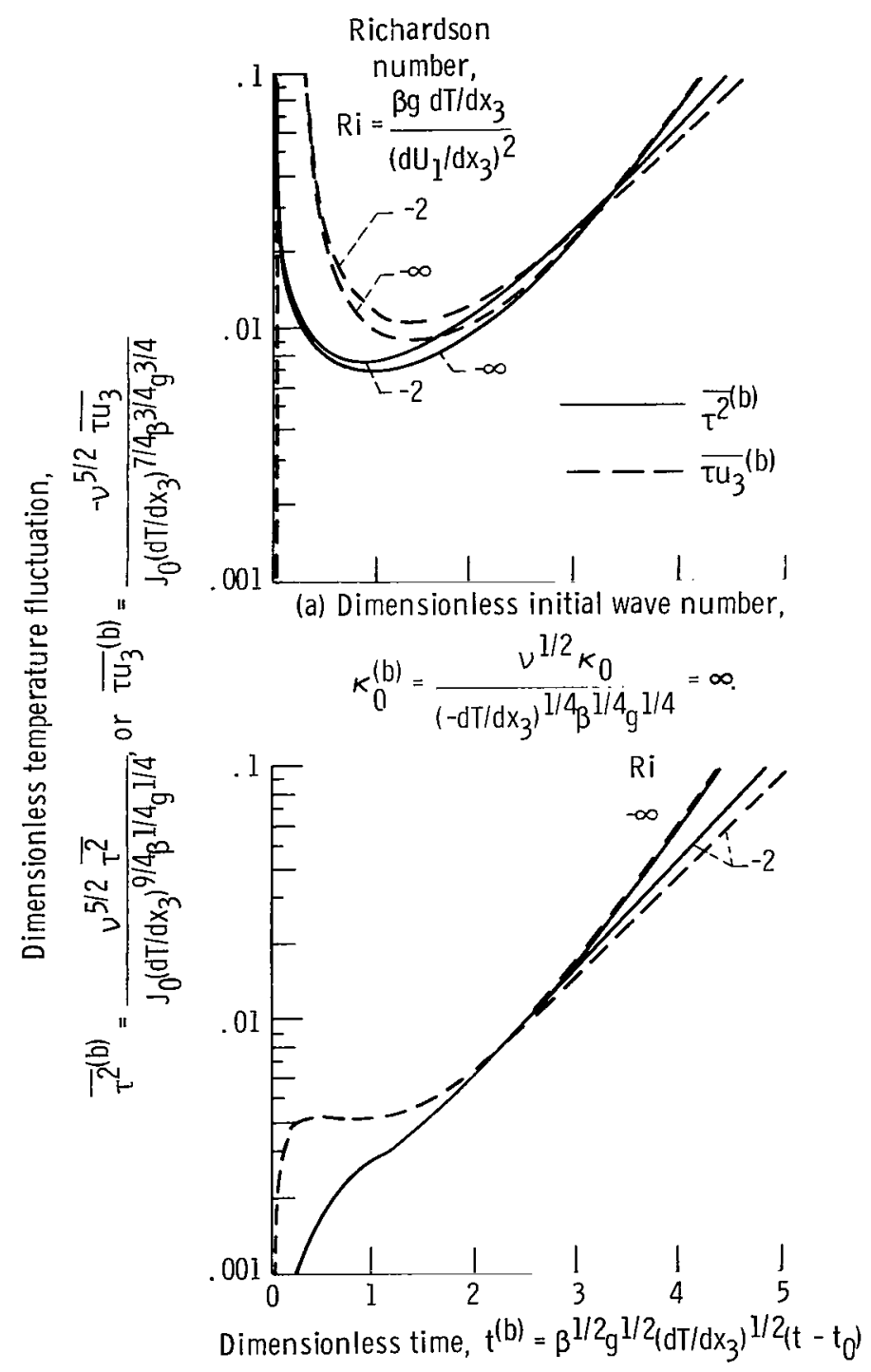

(b) Dimensionless initial wave number,

$$
\text { (b) } \kappa_{0}^{(b)}=\frac{v^{1 / 2} \kappa_{0}}{\left.\left(-d T / d x_{3}\right) 1 / 4_{\beta}\right] / \dot{A}_{g} 1 / 4}=1
$$

Figure 4. - Effect of uniform shear on variation with time of temperature fluctuations and temperature-velocity correlations in flow with destabilizing buoyancy. Prandt। number, 0.7 . 
turbulence is completely controlled by the destabilizing buoyancy forces $(\mathrm{R} i=-\infty)$. Although all turbulent energy components can increase with time when shear is absent, the component in the direction of the buoyancy forces is, in that case, at least an order of magnitude greater than the other components. On the other hand, when both buoyancy and shear are present, all components can be of the same order of magnitude.

Loeffler has recently considered the effect of a gradient in electric charge and an applied electric field on homogeneous turbulence (ref. 6). That problem is analogous to the present one for the case when no shear is present and the Prandtl number is infinite. It was found that the turbulent energy increases without limit as time increases, when the electric field is in the direction of increasing charge density. For large times the turbulent energy was proportional to $\left(\exp t^{(b)}\right) /\left(t^{(b)}\right)^{3}$.

As the shear increases (as $\mathrm{Ri}$ goes from $-\infty$ to -2 ), the turbulent activity in general increases, at least at the earlier times. The shear does not seem to affect $\frac{\tau^{2}}{(b)}$ or $\frac{\tau \mathrm{u}_{3}}{(\mathrm{~b})}$ at the smaller times when $\kappa_{0}^{(\mathrm{b})}=1$, however. At larger times, although $\overline{\mathrm{u}_{1}^{2}}$ and $\overline{\mathrm{u}_{2}^{2}}$ increase with increasing shear, $\overline{\mathrm{u}_{3}^{2}}, \overline{\tau^{2}}$, and $\overline{\tau \mathrm{u}_{3}}$ all decrease with increasing shear. These decreases appear to be related to the fact that at large times the presence of the shear causes energy to be drained out of the $\overline{u_{3}^{2}}$ component (as discussed earlier), and thus out of $\overline{\tau u_{3}}$ and $\overline{\tau^{2}}$ (see eqs. (5) and (6)).

\section{CONCLUDING REMARKS}

Although weak homogeneous turbulence with a uniform shear ultimately decays with time, the presence of destabilizing buoyancy forces in the direction of the mean velocity gradient prevents that decay. In that case the buoyancy forces replenish the energy being drained out of the component of the turbulence in the direction of the mean velocity gradient, and the turbulent energy increases without limit as time increases. Apparently the energy can increase without limit because the effective Reynolds and Rayleigh numbers are infinite in an unbounded fluid. As the scale or mixing length of the turbulence continues to grow, the eddies encounter larger and larger velocity and temperature differences, so that the effective driving forces on the turbulence continue to grow.

Lewis Research Center,

National Aeronautics and Space Administration, Cleveland, Ohio, July 6, 1970, 129-01. 


\section{APPENDIX - SYMBOLS}

a

b

g

$\mathrm{g}_{\mathrm{i}}$

$\mathrm{g}_{3}$

$\mathrm{J}_{\mathrm{O}}$

$\operatorname{Pr} \quad$ Prandtl number, $\nu / \alpha$

p pressure

$\mathrm{Ri}$

$\vec{r}, r_{i}$

$\mathrm{T}$

t

$t_{0}$

$\mathrm{U}_{\mathrm{i}}$

$u_{i}$

$\mathrm{x}_{\mathrm{i}}$

$\alpha$

$\beta$

$\Gamma_{\mathrm{j}}$ $\mathrm{dU}_{1} / \mathrm{dx}_{3}$ $\mathrm{dT} / \mathrm{dx}_{3}$ conditions

pressure

$\mathrm{P}^{\prime}$

mean temperature

time

initial time

space coordinate

thermal diffusivity

expansion coefficient

defined by eq. (12) vertical velocity gradient,

vertical temperature gradient,

vertical body force/unit mass

in $-\mathrm{x}_{3}$-direction (gravitational force/unit mass), $-\mathrm{g}_{3}$

body force component/unit mass

vertical body force/unit mass

constant that depends on initial

Richardson number, $\frac{\beta \mathrm{g} \mathrm{dT} / \mathrm{dx}_{3}}{\left(\mathrm{dU}_{1} / \mathrm{dx}_{3}\right)^{2}}$

vector between points $P$ and

mean velocity component

fluctuating velocity component $\gamma_{i} \quad$ defined by eq. (8)

$\Delta \quad$ defined by eq. (12)

$\delta \quad$ defined by eq. (9)

$\delta_{\mathrm{ij}} \quad$ Kronecker delta

$\theta, \varphi \quad$ angular coordinates

$\kappa_{\mathrm{i}} \quad$ wave number component

$\kappa_{0} \quad$ characteristic initial wave

number (s ee eq. (10))

kinematic viscosity

density

$\tau \quad$ temperature fluctuation

$\bar{\tau} u_{i} \quad$ temperature-velocity correlation

$\varphi_{\mathrm{ij}} \quad$ defined by eq. (7)

$\psi_{i j} \quad$ defined by eq. (12)

Subscripts:

1 in flow direction

3 in vertical direction, which is direction of mean velocity gradient and buoyancy force

Superscripts:

- at point $\mathrm{P}^{\prime}$

- average

(a) parameter nondimensionalized by quantities related to shear

(b) parameter nondimensionalized by quantities related to buoyancy 


\section{REFERENCES}

1. Deissler, Robert G. : Effects of Inhomogeneity and of Shear Flow in Weak Turbulent Fields. Phys. Fluids, vol. 4, no. 10, Oct. 1961, pp. 1187-1198.

2. Fox, J.: Velocity Correlations in Weak Turbulent Shear Flow. Phys. Fluids, vol. 7, no. 4, Apr. 1964, pp. 562-564.

3. Deissler, R. G.: Effect of Initial Condition on Weak Homogeneous Turbulence with Uniform Shear. Phys. Fluids, vol. 13, no. 7, July 1970, pp. 1868-1869.

4. Deissler, Robert G.: Effects of Combined Buoyancy and Shear on Weak Homogeneous Turbulence. NASA TN D-3999, 1967.

5. Deissler, Robert G.: Turbulence in the Presence of a Vertical Body Force and Temperature Gradient. J. Geophys. Res., vol. 67, no. 8, July 1962, pp. 30493062.

6. Loeffler, A. : Electric Field Modification of Turbulence in a Fluid Containing Space Charge. Grumman Research Dept. Rep. RE-384, July 1970. Grumman Aircraft Engineering Corp., Bethpage, N. Y. 


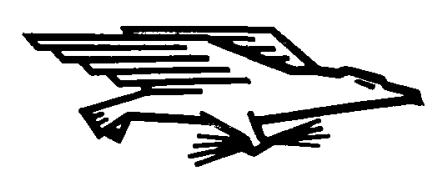

POSTAGE AND FEES PAID NATIONAL AERONAUTICS AND SPACE ADMINISTRATION

ATT E. LOU CCMMAN, CHIEF,THCH. LIBRARY

$\begin{array}{ll}\text { POSTMASTER: } & \text { If Undeliverable (Secrion } 158 \\ \text { Postal Manual) Do Not Return }\end{array}$

"The aeronautical and space activities of the United States shall be conducted so as to contribute. . . to the expansion of buman knowladge of phenomena in the atmosphere and space. The Administration shall provide for the widest practicable and appropriate dissemination of information concerning its activities and the results thereof."

\section{NASA SCIENTIFIC AND TECHNICAL PUBLICATIONS}

TECHNICAL REPORTS: Scientific and technical information considered important, complete, and a lasting contribution to existing knowledge.

TECHNICAL NOTES: Information less broad in scope but nevertheless of importance as a contribution to existing knowledge.

TECHNICAL MEMORANDUMS:

Information receiving limited distribution because of preliminary data, security classification, or other reasons.

CONTRACTOR REPORTS: Scientific and technical information generated under a NASA contract or grant and considered an important contribution to existing knowledge.
TECHNICAL TRANSLATIONS: Information published in a foreign language considered to merit NASA distribution in English.

SPECIAL PUBLICATIONS: Information derived from or of value to NASA activities. Publications include conference proceedings, monographs, data compilations, handbooks, sourcebooks, and special bibliographies.

\section{TECHNOLOGY UTILIZATION} PUBLICATIONS: Information on technology used by NASA that may be of particular interest in commercial and other non-aerospace applications. Publications include Tech Briefs, Technology Utilization Reports and Notes, and Technology Surveys.

Details on the availability of these publications may be obtained from:

SCIENTIFIC AND TECHNICAL INFORMATION DIVISION

NATIONAL AERONAUTICS AND SPACE ADMINISTRATION

Washington, D.C. 20546 